\title{
EFECTO DEL DETERGENTE BIODEGRADABLE (Aquil Sulfonato de Sodio) EN EL CONSUMO DE OXÍGENO Y TASA DE FILTRACIÓN DEL BIVALVO Semimytilus algosus
}

\section{EFFECT OF BIODEGRADABLE DETERGENT (Alquil Sulfonate of Sodium) ON THE OXYGEN CONSUMPTION AND FILTRATION RATES OF BIVALVE Semimytilus algosus}

\author{
Guillermo Álvarez' ${ }^{*}$, Graciela Medina ${ }^{2}$, Guadalupe Sánchez ${ }^{2}$
}

\section{RESUMEN}

El consumo de oxígeno específico (COE) e individual (COI) de Semimytilus algosus $(0,0856$ a 0,1869 g) fue determinado en respirómetros cerrados estáticos $(300 \mathrm{ml}$ aprox.) en presencia de 3 concentraciones de detergente $\left(\mathrm{C} 0=0,00 ; \mathrm{C} 1=0,02 ; \mathrm{C} 2=0,04 ;\right.$ y $\mathrm{C} 3=0,06 \mathrm{mg} \mathrm{l}^{-i}$ de detergente). Para detectar el oxígeno disuelto se usó el método de Winkler. La tasa de Filtración individual (TF!) fue calculada en ausencia de detergente por consumo de la diatomea Chaetoceros gracilis con los mismos individuos expuestos al detergente durante los experimentos de respiración. Las condiciones de experimentación fueron $35 \%$ de salinidad y $17 \pm 0,5^{\circ} \mathrm{C}$. Los resultados observados indican que el detergente aún siendo biodegradable, a tiempos cortos de exposición altera la tasa respiratoria e incrementa el consumo de oxígeno y velocidad de la tasa de filtración a medida que la concentración de detergente se incrementa.

Palabras claves: Consumo de oxigeno, Tasa de filtración, Alquil Sulfonato de sodio, Semimytilus algosus

\section{ABSTRACT}

The specific (COE) and individual (COI) oxygen consumption of Semimytilus algosus $(0,0856$ to $0,1869 \mathrm{~g}$ ) were determinated in static closed chambers ( $300 \mathrm{ml}$ aprox.), using 3 concentrations of detergent ( $\mathrm{C} 0=0,00 ; \mathrm{C} 1=0,02 ; \mathrm{C} 2=0,04 ;$ and $\mathrm{C} 3=0,06 \mathrm{mg} \mathrm{l}^{-1}$ of detergent). To detect the dissolved oxygen was used the Winkler method. The filtration rate was determinated without detergent by filtration of Chaetoceros gracilis, with the same individuals as were exposed to the detergent during the respiration experiments. The experiment conditions were $35 \%$ of salinity and $17 \pm 0,5{ }^{\circ} \mathrm{C}$. The results indicated that the detergent, even though it is biodegradable, at short periods of exposition disturbs the respiration rate increasing the oxygen consumption and velocity of filtration rate with the increase of detergent concentration.

Key words: Oxygen consumption, Filtration rates, Alkil sulfonate of sodium, Semimytilus algosus

\section{INTRODUCCIÓN}

La contaminación marina producida por las

\footnotetext{
" Correspondencia: Laboratorio de Reproducción y Biología del Desarrollo. Facultad de CC. BB. UNMSM Av. Venezuela $s / n$, Lima, Perú. alvarez.g@usa.net d190113@unmsm.edu.pe 2 Área de Ecofisiología Acuática, Dirección General de Investigaciones Oceanográficas, IMARPE, Esq. Gamarra y Valle, Callao, Perú. gsanchez@imarpe.gob.pe
}

actividades domésticas e industriales constituye una de las fuentes de contaminación más agresivas en nuestros días. Entre los diversos contaminantes se encuentran los detergentes domésticos, sean éstos biodegradables o no. Los detergentes biodegradables se caracterizan por presentar una cadena carbonada no ramificada, con lo que facilitan la biodegradación por los microorganismos y disminuyen el efecto tóxico en la biota acuáfica (Perraza 1998). A pesar de esto, es importante el tiempo de inicio del proceso de biodegradación, pues si éste es lo suficiente- 
Tabla 1. Peso seco, consumo de oxígeno individual (CIND) y específico (CESP), desviación estándar (DE) y coeficiente de variación (CV) de S. algosus.

\begin{tabular}{|c|c|c|c|c|c|c|c|c|c|}
\hline \multirow{2}{*}{$\begin{array}{l}\text { Conc. de } \\
\text { Deterg. }\end{array}$} & \multicolumn{3}{|c|}{$\begin{array}{c}\text { Peso Seco } \\
\text { PS } \\
\end{array}$} & \multicolumn{3}{|c|}{$\begin{array}{c}\text { Consumo Individual } \\
\text { CIND }\end{array}$} & \multicolumn{3}{|c|}{$\begin{array}{c}\text { Consumo Específico } \\
\text { CESP }\end{array}$} \\
\hline & $\begin{array}{c}\text { Media* } \\
\mathrm{g}\end{array}$ & $\mathrm{DE}$ & $\mathrm{CV}$ & Media" & $\mathrm{DE}$ & $\mathrm{CV}$ & Media* & $\mathrm{DE}$ & $\mathrm{CV}$ \\
\hline$\infty 0$ & 0,142 a & 0,017 & 12,1 & 0,196 a & 0,008 & 3,96 & 1,393 a & 0,185 & 13,3 \\
\hline $\mathrm{C} 1$ & 0,127 a & 0,024 & 18,9 & $0,3 i 1 b$ & 0,016 & 5,14 & $2,499 \mathrm{~b}$ & 0,389 & 15,6 \\
\hline $\mathrm{C} 2$ & 0,155 a & 0,03 & 19,5 & $0,350 \mathrm{c}$ & 0,022 & 6,12 & $2,303 \quad b$ & 0,317 & 13,7 \\
\hline $\mathrm{c3}$ & $0,129 \mathrm{a}$ & 0,019 & 15,4 & $0,220 \mathrm{a}$ & 0,014 & 6,37 & $1,867 \mathrm{~b}$ & 0,199 & 7,56 \\
\hline
\end{tabular}

*Letras iguales no muestran diferencia estadística significativa $(p<0,05) \mathrm{CO}=0,00, \mathrm{C1}=0,02$ $\mathrm{C} 2=0,04$ y $\mathrm{C} 3=0.06 \mathrm{mgl}^{-1}$ de detergente, respectivamente. $\mathrm{CIND}=\mathrm{mgO}_{2} I^{-1} \mathrm{ind}^{-1} \mathrm{~h}^{-1}, \mathrm{CESP}=$ $\mathrm{mgO}_{2} \mathrm{l}^{-1} \mathrm{~g}^{-1} \mathrm{~h}^{-1}$.

mente largo causaría efectos subletales.

Los efectos de los tóxicos en las comunidades acuáticas son determinados mediante bioensayos de toxicidad letal y subletales a condiciones controladas de laboratorio (Caims y Niederlenner 1994) utilizando organismos modelo, que tengan características tales como el ser de amplia distribución y susceptibles a los tóxicos.

El consumo de oxígeno y la tasa de filtración son dos de los procesos físiológicos que permiten establecer el estado metabólico en los invertebrados marinos (Giffiths 1980; Diehl et al. 1986; Gómez et al. 1995; Phan e? al. 1993). Estos procesos fisiológicos son ampliamente usados para detectar alteraciones metabólicas en estos organismos al ser expuestos a variaciones de factores exógenos, tales como temperatura o salinidad, así como efectos de tóxicos (Bayne 1973; Chen y Lai 1993; Donald et al. 1993; Vernberg y Vernberg 1972).

El presente estudio tiene por objetivo determinar el grado de efecto crónico o subletal de un detergente doméstico biodegradable en el consumo de oxígeno y la tasa de filtración del molusco bivalvo Semimytilus algosus, que es un modelc adecuado nara la evaluación de tóxicos, pues presenta una amplia distribución y abundancia en el iitoral peruano.

\section{MATERIAL Y MÉTODOS}

Los individuos utilizados fueron de la especie S. algosus "chorito", molusco bivalvo

Tabla 2. Tasa de filtración individua! (TFl), desviación estándar (DE) y coeficiente de variación (CV) de $S$. algosus.

\begin{tabular}{|c|c|c|c|}
\hline \multirow{2}{*}{$\begin{array}{c}\text { Concentración de } \\
\text { Detergente. }\end{array}$} & \multicolumn{3}{|c|}{ Tasa de Elltración (TFI) } \\
\hline & $\begin{array}{c}\text { Media } \\
\mathrm{m}^{*} \mathrm{~h}^{-1} \text { ind }^{-1} \\
\end{array}$ & $D E$ & $\mathrm{CV}$ \\
\hline $\mathrm{CO}$ & 91,9280 a & $50.8 \leadsto$ & 55,3 \\
\hline $\mathrm{Cl}$ & $118,2770 \mathrm{a}$ & 36,46 & 30,8 \\
\hline$c 2$ & $189,9490 \mathrm{~b}$ & 54,35 & 28,6 \\
\hline $\mathrm{C3}$ & 102.55302 & 29.63 & 28.9 \\
\hline
\end{tabular}

*Letras iguales no muestran diferencia estadística significativa $(p<0,05) C 0=0,00, C 1=0,02$ $\mathrm{C} 2=0,04$ y $\mathrm{C} 3=0,06 \mathrm{mg} \mathrm{l}^{-1}$ de detergente, respectivamente. 


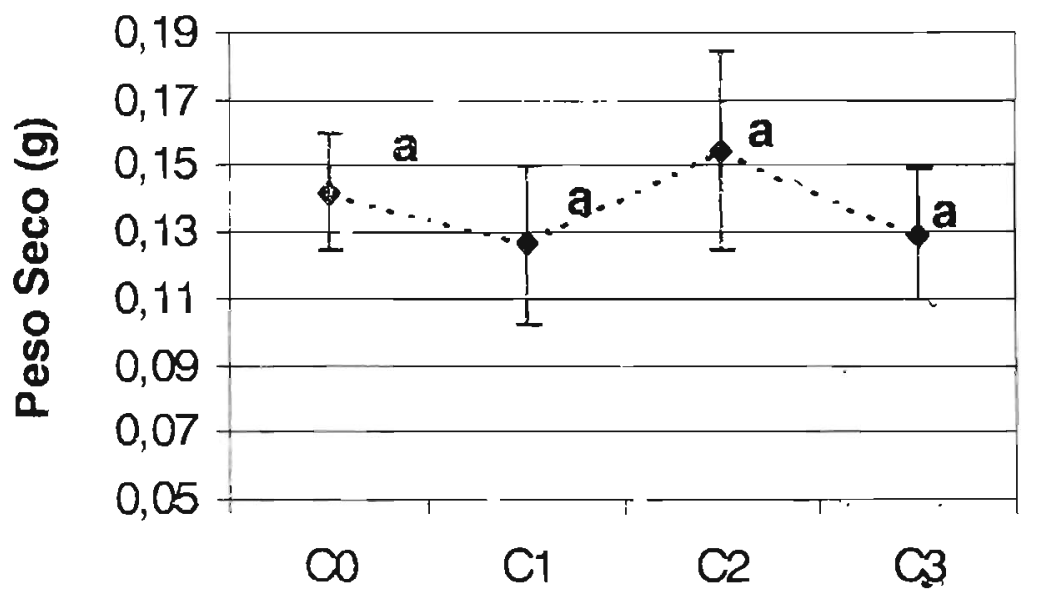

\section{Detergente $\left(\mathrm{mg} \mathrm{l}^{-1}\right)$}

Figura 1. Peso Seco (PS) de S. algosus a $\mathrm{CO}=0,00 ; \mathrm{C} 1=0,02 ; \mathrm{C} 2=0,04 ; \mathrm{C} 3=0,06 \mathrm{mg} \mathrm{l}^{-1} \mathrm{de}$ detergente. (Las letras iguales indican que no existe una diferencia estadístiça significativa ( $p$ $<0,05)$.)

que habita la zona intermareal rocosa. Se utilizaron individuos con un peso seco (PS) medio de $0,134 \pm 0,03 \mathrm{~g}(\mathrm{CV}=19,2)$ (Tab. y Fig. 1), fueron colectados en la Isla San Lorenzo (Callao) (77 $12^{\prime}-12^{\circ} 05^{\prime}$ ) en el mes de febrero de 1999. Estos individuos fueron mantenidos por espacio de una semana en agua de mar filtrada (filtro de $1 \mathrm{~mm}$ ), $17 \pm 0,5^{\circ} \mathrm{C}$ de temperatura y $35 \%$ de salinidad con aireación constante. Durante el periodo de estudio, $S$. algosus fue alimentado dos veces por día con la diatomea Chaetoceros gracilis.

El detergente doméstico utilizado contiene el tensoactivo biodegradable Alquil

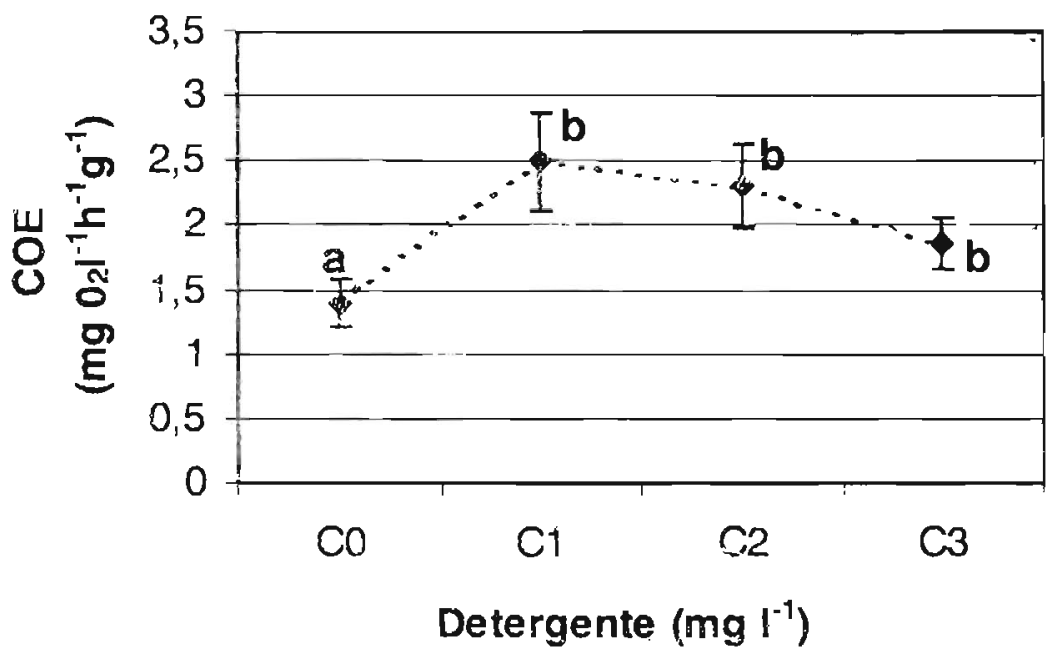

Figura 2. Consumo de oxígeno específico (COE) de $S$. algosus a $C 0=0,00 ; C 1=0,02 ; C_{2}=$ 0,$04 ; \mathrm{C} 3=0,06 \mathrm{mg} \mathrm{I}^{-1}$ de detergente. (Las letras iguales indican que no existe diferencia estadística significativa $(p<0,05)$.) 


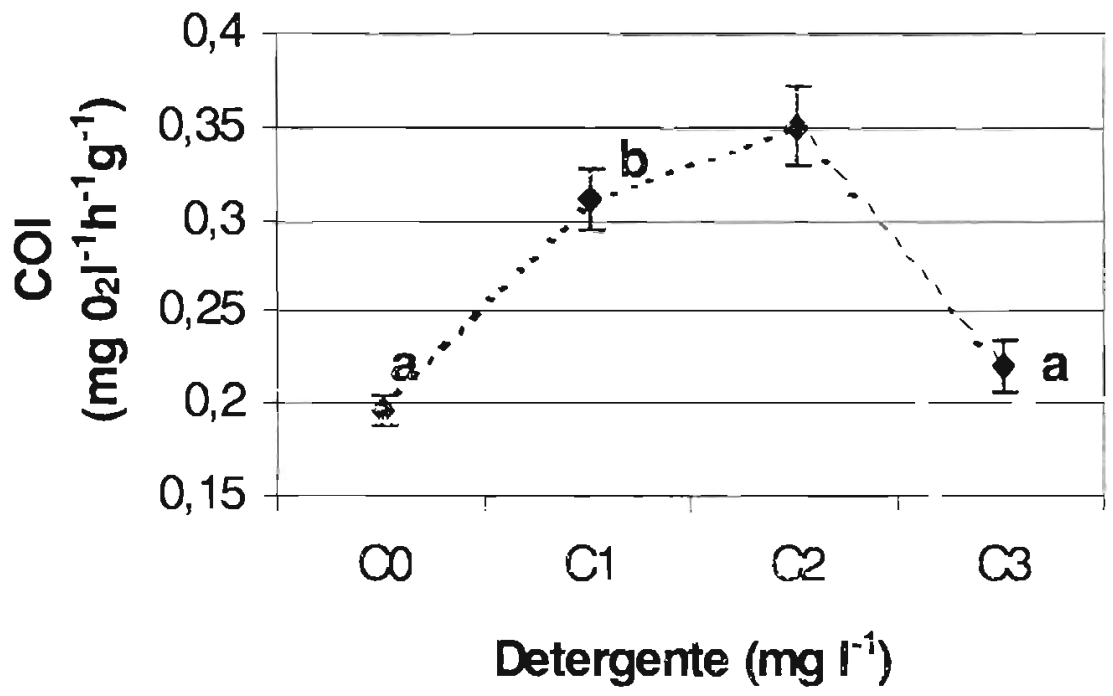

Figura 3. Consumo de oxígeno individual $(\mathrm{CO})$ de $S$. algosus a $\mathrm{CO}=0,00 ; \mathrm{C}_{1}=0,02 ; \mathrm{C} 2=$ 0,$04 ; \mathrm{C} 3=0,06 \mathrm{mg} \mathrm{l}{ }^{-1}$ de detergente. (Letras iguales no muestran diferencia estadística significativa $(p<0,05)$.)

Sulfonato de Sodio, que corresponde a los detergentes llamados blandos o LAS (Alquilbenceno-sulfonato de sodio, Blando = LAS) (Peraza 1998). El experimento fue realizado con tres concentraciones de detergente: $\mathrm{C} 1=0,02, \mathrm{C} 2=0,04$ y C3 $=0,06 \mathrm{mg} \mathrm{l}^{-1} \mathrm{de}$ detergente respectivamente. Estas concentrâciones fueron determinadas por experimentos previos, teniendo como concentración máxima de referencia el valor del LC50 (1-2,5 mg $\left.1^{-1}\right)$ para Odontesthis regia regia «pejerrey», determinado en el ADECO-IMARPE.

El diseño experimental planteado fue: análisis de varianza completamente aleatorio ( $P$ $<0,05$ ) y contraste de medias Duncan (Soká! y Rohlf 1962). Para los análisis se utilizó el software STATGRAPHIC v. 0.7.

\section{Tasa respiratoria}

\section{(Consumo de oxígeno)}

Para los ensayos de respiración se utilizaron como cámaras respirométricas recipientes cilíndricos con tapa esmerilada de un volumen aproximado de $300 \mathrm{ml}$. Para cada concentración de detergente, incluyendo la con- centración $\mathrm{C} 0$ (sin detergente), se dispusieron cuatro respirómetros por cada concentración, tres con un animal cada uno y un respirómetro sin animal.

Los organismos utilizados fueron mantenidos en ayunas 24 horas antes de los ensayos de respiración y filtración, esto para evitar que el consumo de oxígeno debido al proceso de digestión enmascare la respiración en estado de reposo (Leite 1997; Armitage y Wall 1982).

Los moluscos fueron mantenidos en los respirómetros por 1 h y $30 \mathrm{~min}$ (tiempo de aclimatación determinado por experimentos previos) para permitir que el estrés causado por el volumen del respirómetro y la rnanipulación de experimentación disminuya. Durante el tiempo de aclimatación los respirómetros se mantuvieron abiertos para facilitar el flujo de agua a temperatura constante $\left(17 \pm 0,5^{\circ} \mathrm{C}\right)$. Al cabo del tiempo de aclimatación se procedi6 a colocar alícuotas de detergente a cada envase para obtener las concentraciones deseadas, e inmediatamente los envases fueron cerrados. El tiempo de respiración fue de 1 h y $30 \mathrm{~min}$, a la misma temperatura y sin aireación.

Fue determinado el Consumo de Oxígeno 


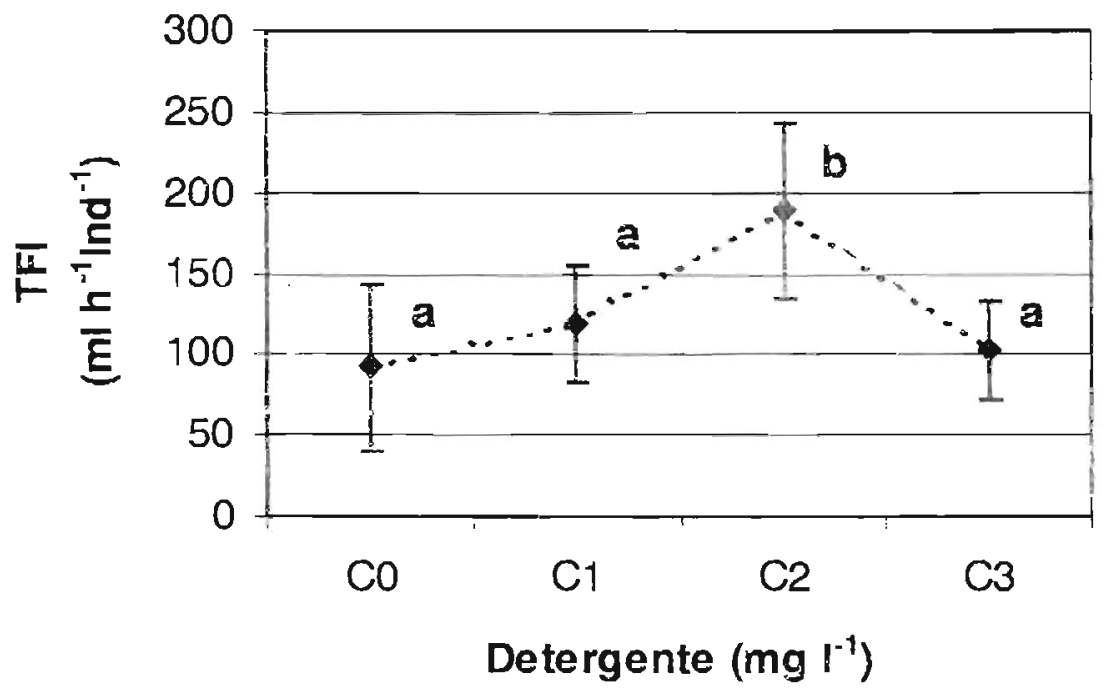

Figura 4. Tasa de filtración individual (TFI) de $S$. algosus, a $C 0=0,00 ; C 1=0,02 ; C 2=0,04$; $\mathrm{C} 3=0,06 \mathrm{mg} \mathrm{I}^{-1}$ de detergente. (Letras iguales no muestran diferencia estadística significativa $(p<0,05)$.)

Individua! (COI), expresado en $\mathrm{mg} \mathrm{O} 2^{1^{-1} \mathrm{~h}^{-1}}$ ind $^{-1}$, y el Consumo de Oxígeno Específico (COE), expresado en $\mathrm{mgO}_{2} \mathrm{l}^{-1} \mathrm{~h}^{-1} \mathrm{~g}^{-1}$ (gramos de PS del molusco). El PS fue determinado a $60^{\circ} \mathrm{C}$ por 48 horas al finalizar las pruebas de respiración y filtración.

El consumo de oxígeno se determinó por la diferencia entre el oxígeno disuelto (OD) de los respirómetros con animal y los respirómetros sin animal. El OD fue determinado por el método químico de Winkjer modificado (ISO-5813 1982).

\section{Tasa de filtración individual}

Al finalizar el tiempo de respiración los organismos fueron sometidos a la prueba de filtración, en ausencia de detergente, manteniendo la identificación correspondiente de cada concentración de detergente.

La Tasa de Filtración Individua! (TFI) se determinó por la velocidad de consumo (filtración) de la diatomea $C h$. gracilis en condiciones controladas. Se utilizó recipientes cilíndricos de $300 \mathrm{ml}$ aproximadamente con un volumen algal de $250 \mathrm{ml}$. Los ensayos fueron a iemperatura $17 \pm 0,5^{\circ} \mathrm{C}$ y leve aireación constante. La concentración algal inicial fue de $28,08 \times 10^{5}$ cel $\mathrm{ml}^{-1}$, y se realizó conteos por triplicado en la cámara de Newbauer para determinar las densidades algales inicial y finales de cada unidad experimental.

La fórmula utilizada para calcular la TFI fue la siguiente (Griffith 1980):

$$
\begin{aligned}
& \text { Tasa de } \\
& \text { Filtracióe }\left(\mathrm{mb}^{-1}\right) \\
& \text { Individual }
\end{aligned}=\frac{\log _{\mathrm{a}} N_{2}-\log _{\mathrm{a}} N_{l}}{\mathrm{~T}} \times \mathrm{V}
$$

\section{RESULTADOS Y DISCUSIÓN}

Los individuos sometidos a las concentraciones de detergente $\mathrm{C} 1, \mathrm{C} 2$ y $\mathrm{C} 3$ no mostraron diferencia significativa pero fueron significativamente más elevados que $\mathrm{CO}$ para el COE. Para el COI, C0 y C3 no mostraron diferencia significante, y evidenciaron ser inferiores a $\mathrm{C} 1$ y $\mathrm{C} 2$, que a su vez mostraron diferencia estadística $(P<0,05)$ (Tab. 1, Fig. 2 y 3). Se resalta que en los individuos expuestos a C2 se observa un COI (Fig. 3) ligeramente más elevado que para $\mathrm{C} 1$ mostrando un efecto del peso individual, pues a pesar de 
no mostrar diferencias signifícátivas peso especificas de los individuos utilizados, los correspondientes a C2 muestran una leve superioridad y mayor variabilicad $(\mathrm{CV}=19,53)$ (Tab. 1 y Fig. 1).

La TFI presenta una diferencia estadística significativa $(P<0,05)$ entre los organismos correspondientes a la concentración de detergente $\mathrm{C} 2$ en comparación con los de $\mathrm{CO}, \mathrm{C} 1$ y $\mathrm{C} 3$, quienes no mostraror diferencia álguna $(\mathrm{P}<0,05)$ (Tab. 2 y Fig. 4).

Estos resultados indican que este molusco sufre alteración en la TFI, probablemente por acumulación de detergente, esto por el razonamiento de que los individuos de la concentración $\mathrm{C} 2$ durante el tiempo de respiración estuvieron expuestos por menos tiempo al detergente que los individuos de la concentración $\mathrm{C} 3$, pero con mayor concentración que jos de C1 (Tab. 1 y 2).

Los resultados por unidad de PS nos sugieren, que $S$. algosus presenta baja sensibilidad a la presencia de detergente, puesto que el COE más elevado fue observado en la menor concentración, a pesar de no ser significante la diferencia (Tab. 1 y Fig. 3). Esto indica que la velocidad de cierre de las valvas se incrementa a medida que la concentración de detergente aumenta, io que es resaltado en los resultados del COI, pues en la mayor concentración (C3) se observó el menor consumo de oxígeno. El cierre de valvas, característica de los moluscos bivalvos, dificulta visualizar el efecto de los tóxicos, como los detergentes, pues los aísla del medio externo y facilita el control de su metabolismo (Gerdes 1983; Schmidt-Nielsen 1990).

La menor sensibilidad de los organismos del intermareal rocoso en comparación cor les organismos en contacto directo con el bentos marino es resa!tando por Bressan et al. (1989), quien comenta que esta sensibilidad se debe al esta: expuestos directamente a efluentes $y$ material particulado en suspensión precipirado en esta capa.
E! efecto tóxico de los detergentes reside en la unión de agentes receptores químicos con los receptores celulares (Perraza 1998, Bressan et al. op. cit.) a nivel de las estructuras branquiales que tienen por función principal el intercambio gaseoso (oxígeno, amoniaco, etc.) y la ingestión de alimento (microalgas) (Schmidt-Nielsen op cit.); de este modo los agentes químicos causan irritación de los tejidos branquiales y dificultan sus funciones. Los resultados observados indican que poí exposición al detergente los tejidos jranquiales realizan secreción de mucus en exceso. Esta secreción incrementaría el consumo de oxígeno (Jorgensen 1981; Colt y Armstrong 1981), en tanto que por comprometimiento de los cilios branquiales y por acumulación de detergente, aumentaria is velocidad de filtración, puesto que el volumen de alimento apto para los moluscos es en parte determinado por el volumen de agua transportada a través de sus branquias y en parte por la acumulación de partículas (Bonadonna et. al. 1990; Griffiths 1980).

\section{CONCLUSIONES}

Las respuestas obtenidas de los organismos expuestos al detergente permiten concluir que el detergente biodegradable utilizado en el presente estudio causa alteraciones en la respiración de este molusco bivalvo incrementando el consumo de oxígeno individual y específico, y en el caso de la tasa de Fitración individual la alteración sería por aceíeración del flujo de filtración a medida que se incrementa el tiempo y concentración de detergente.

Por otra parte, los detergentes, a pesar de ser biodegradables, causan efectos significativos en el COE, COI y TFI de los organismos a ellos expuestos, aun en periodos cortos de exposición, antes de permitir que el proceso de biodegradación comience. 
Agradecimientos: Nuestro agradecimiento al Blgo. Víctor Hugo Vera y al técnico Edwin Pinto por el apoyo y las facilidades brindadas para la ejecución del presente trabajo, así como a la Blga. Giovana Vera. Los experimentos fueron realizados en las instalaciones del Área de Ecofisiología Acuática (ADECO) de la Dirección General de Investigaciones Acuáticas del Instituto del Mar del Perú (IMARPE).

\section{LITERATURA CITADA}

Armitage, K. B. y T. J. Wall. 1982. The effects of body size, starvation and temperature acclimatization on oxygen consumption of the cray fish Orconectes nais. Comp. Biochem. Physiol. 73A(1): 63-68.

Bayne, B. L. 1973. Physiological changes in Mytilus edulis induced by temperature and nutritive stress. J. Mar. Biol. Ass. UK 53: 39-58.

Bonadonna, L.; L. Voltera; F.A. Aulicino y L. Mancini. 1990. Accululation power of some bivalve molluscs. Marine Pullution Bulletir 21(2):8184.

Bressan, M.; R. Brunetti; S. Casellato; G.C. Fava; P. Giro; P. Marin; Negrisolo; L. Tallarini; S. Thomann; L. Tosoni; M. Turchetto y G.C. Campesan. 1989. Effects of linear alkylbenzene sulfonate (LAS) on benthic organims. Tenside Surfactant Detergent 26(2): 147-158.

Cairns, J. Jr. y B.R. Niederlehner. 1995. Ecologycal toxicity testing. Scale, Complexity and Relevance. Lewis Publishers, USA. 228 pp.

Cehn, J. C. y S.H. Lai. 1993. Effects of temperature and salinity on oxygen consumption and ammonia$\mathrm{N}$ excretion of juvenile Penaeus japonicus bate. J. Exp. Mar. Biol. Ecol. 165:161-170.

Colt, J. E. y D.A. Armstrong. 1981. Nitrogen Toxicity to crustaceans, fish and Molluses. In: Allen and Kinney eds. Proceedings of the BioEngineering Symposium for fish culture. pp.
34-47.

Diehl, J. W.; P.M. Gaffney y R.K. Koehn. 1986. Physiological and Genetics aspects of growth in the mussel Mytilus edulis. 1. Oxygen consumption, growth, and weight loss. Physiol Zool. 59(2):201-211.

Donald, J. R.; P.S. Oshida; A.J. Meams y T.C. Ginn. 1993 Effects of pollution on saltwater organisms. Water Emvmt. Resh. 65(4):573584.

Gerdes, D. 1983. The paciphic oyster Crassostrea gigas Part II: Oxygen consumption of larvae and adults. Aquaculture, 31:221-231.

Gomes, V.; N. Phan y M.J. Passos. 1995. Estudo do metabolismo de rotina e excreçăo de amonia do anfípoda antártico Waldeckia obesa em duas temperaturas distintas. Bolm. Inst. Oceanogr. São Paulo. 43(2):129-139.

Griffiths, R. J. 1980. Filtration, respiration and assimilation in the black mussel choromytilus meridionalis. Marine Ecology Prog. Ser. 3:6370 .

Ĩinternational Standard (ISO), 1983. Water qualityDetermination of dissolved oxygenIodometric method. International Organization for Standardization, ISO-5813.6 pp.

Jorgensen, C. B. 1981. Feeding and Cleaning mechanisms in the suspension feeding Bivalvia Mytilus edulis. Marine Biology 65:159-163

Lite, M. S. 1997. Estudo bioenergético do peixe-rei Xenomelaniris brasiliensis (QUOY \& GAIMARD 1924) (PICES, ATHERINIDAE). São Paulo: USP, 1997. 154p. Dissertação (Mestrado em Oceanografia Biológica)-Instituro Oceanográfico, Universidade de Săo Paulo.

Peraza, A. 1998. Los detergentes. CEPIS. Doc. 3400/ P45/12894. pp. 1-4.

Phan, N.; V. Gomes; D.M. Morais y M.J. Passos. 1993. Estudo bioenergético de animais marinhos costeiros. I: Paralonchurus brasilensis (Perciformes, Sciaenidae). Bolm. Inst. Oceanogr. São Paulo. 10:199-215.

Scrimidt-Nielsen, K. 1990. Animal physiology: Adaptation and environment. Fourth Edition. Cambridge University Press, USA. 602 pp.

Soka! R. R. y F.J. Rohlf. 1962. Biometry: The principles and practice of statistics in biological reseach. W. H. Freeman Company, United States of América. $776 \mathrm{pp}$.

Vernberg, W. B. y J. Vernberg. 1972. The synergistic effects of temperature, salinity and mercury on survival and metabolism of the adult fiddler crab, Uca pugilator. Fish. Bull. 70:415-420. 